the anterior groove, but the dorsal surface of the second and third rows of phalanges being in contact with the upper part of the anterior wall of the uterus. Steady pressure upwards and backwards here produced a corresponding amount of reinversion anteriorly. In changing the hand from the posterior to the anterior aspect of the uterus, care was taken to keep up pressure on the fundus by any part of the hand or wrist which happened to be available for the purpose, in order to prevent a recurrence of the inversion which had already been corrected. This process was repeated once or twice till the fundus uteri was just a little below the anterior and posterior grooves; when under continued pressure upon the fundus uteri itself with the dorsal surface of the first row of phalanges, complete reinversion of the uterus occurred rather suddenly. While reinversion was being effected counter pressure with the left hand on the abdomen was applied. The time occupied in separating the placenta and effecting complete reinversion was about 20 minutes.

The patient's general condition was now attended to, strychnine hypodermically, brandy in large quiantity, and a saline enema being given, and hot bottles used. On the fourth day there was a sharp attack of septic intoxication which subsided by the tenth day, and on the eleventh day an attack of hrmatemesis occurred. The patient then made an uninterrupted recovery, the anæmia gradually disappearing under the influence of iron. During convalescence the uterus was found to be deviated to the left.

This patient has since had several children without any complications occurring, showing that no permanent illeffects were left by the accident.

The following points may be noted. 1. The patient was previously very much run down and anæmic. 2. She was a primipara. 3. The placenta was attached to the fundus uteri and was pathologically adherent. 4. The uterus did not contract very satisfactorily as long as the placenta remained attached. 5. The child was of large size. 6. The patient "bore down" considerably while partly under chloroform and while the uterus was relaxed.

It may be that traction upon the cord produces in such a case a temporary paresis of the uterine muscle at the placental site and for a short distance around it, and thus predisposes to inversion. This point, I think, has not been investigated.

The hæmorrhage in this case was quite insufficient to account for the extreme and prolonged collapse. Collapse is most marked when the accident happens suddenly, and seems to be due to strangulation of the inverted uterus, and to traction upon the Fallopian tubes, round ligaments, ovaries, and ovarian ligaments, with possibly slight laceration of peritoneum.

This accident is fortunately of very uncommon occurrence. It is supposed to happen once in about 30,000 cases. This is the only case I have seen in an obstetrical practice of considerably over 2000 cases. I have waited a long time before reporting this case, and as yet no similar train of circumstances has arisen in my experience. The notes of the case were taken when it occurred. The hæmatemesis was post-operative, and of this form of hæmatemesis I have only had one other case in obstetric practice. Broadstairs, Kent.

\section{CASE OF EXTENSIVE FIBRO-ANGIOMA TREATED BY RADIUN.}

BY F. H. JACOB, M.D. LoND., M.R.C.P. LOND.,

HONORARY PHYSICIAY TO THE HOSPITAT FOR SICK GHILDREY, NOTTINGHAM, AND TO THE NOTTINGHAM GENERAL HOSPITAL,

A Boy, 8 months old, was admitted to hospital on Dec. 31st, 1910 , for a large growth on the chest, which had been first noticed on August Bank Holiday. It had grown rapidly of late. Extending over the anterior portion of the chest from the clavicles to the diaphragm and from mid-axilla to midaxilla, was a large raised area of the colour of raw beefsteak, which colour could only partially be removed by pressure; on the surface were minute telangiectases. On examination the skin was felt to be very markedly indurated and much thickened, and it felt as though the subcutaneous tissues were also affected; the margin was sharply defined and raised about half an inch above the surrounding skin. The whole mass was flxed and it was only possible to move it slightly on the chest wall.
There was considerable discussion as to whether it was a sarcoma or some unusnal variety of angioma. A small portion was removed and sections cut.

Professor S. G. Shattock very kindly gave us an opinion on the sections and pronounced it to be a fibro-angioma. It

FIG. 1.

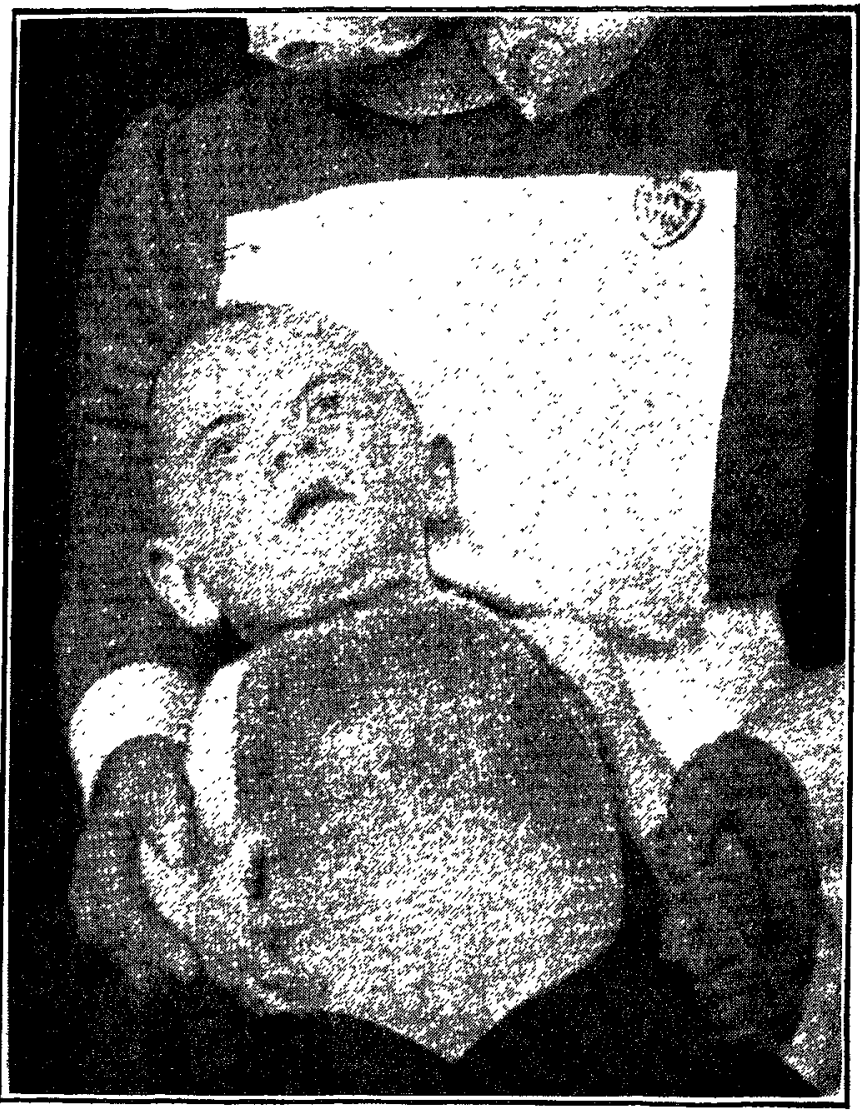

Patient before treatment.

FIG. 2.

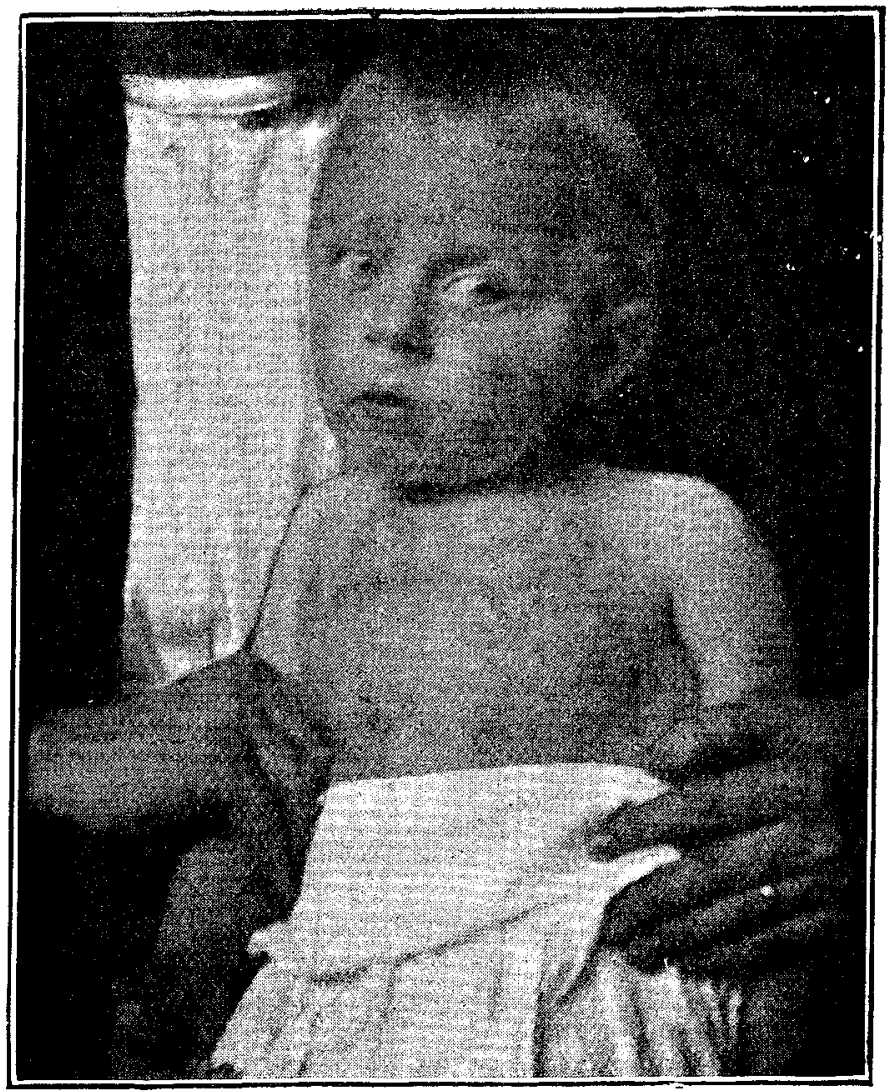

Patient after treatment.

was treated at first by electrolysis and later by radium. It has now completely disappeared. There were also several small nodules on the body, all subcutaneous, and one does not know if they were of the same nature, but they have all disappeared without any treatment. Figs. 1 and 2 show the case before treatment and at the present time.

Nottingham. 


\section{A NEW FORM OF TUBERCULIN (T.F.) SOME NOTES ON ITS DIAGNOSTIC AND THERAPEUTIC VALUE. \\ BY W. C. LYONS, M.B., CH.B. EuIN.,}

SENIOR ASSISTANT MEDICAL OFFICER, UNION HOSPITAL, BRADFORD.

Is applying the various tests with old tuberculin for the diagnosis of tuberculosis one is struck by the comparatively large number of patients reacting positively in whom no trace of tuberculosis is found clinically, and who give no history of a tubercular infection.

In a series of 15 post-mortem examinations on patients who at times varying from one to two months before death gave a positive reaction, both with the vaccination and blister methods of applying old tuberculin for diagnosis, six were found to be tubercular subjects. Of these, three proved to have pulmonary tuberculosis, while one had lesions of tubercular peritonitis, the lungs being healthy. In one case a healed lesion was demonstrated at one apex, and in another caseous glands of the neck were found. In the remaining nine cases no obvious tubercular disease was discernible, special attention being directed to the bronchial, mediastinal, and mesenteric glands, lungs, and meninges.

One case which yielded a positive reaction with both the vaccine inoculation and blister tests was very instructive. A male, aged 46, was admitted suffering from pulmonary tuberculosis. The usual clinical symptoms were present, but tubercle bacilli were never demonstrated in the sputum on repeated examination. On examination the right lung presented a localised area of dulness, with moist sounds and a prolonged expiratory murmur. It was accordingly diagnosed as phthisical. The condition rapidly changed, and a few weeks later, on development of further signs, malignant disease of the lungs was suspected. At the necropsy this view was confirmed. The right lung and bronchial glands were found to be infiltrated with areas of carcinoma, and, further, no obvious trace of tuberculous disease was found.

In investigating this subject it was thought that old tuberculin might contain some substance which produced a positive reaction, perhaps by irritation, on apparently healthy individuals, whose skin is not sensitised by the products of the tubercle bacillus, and if that substance were removed a positive reaction would more definitely indicate the presence of tubercular disease.

On adding to 10 c.c. of old tuberculin 60 c.c. of absolute alcohol a cloudiness appears in the solution, which on standing for 24 hours settles as a precipitate which clings to the sides of the vessel. The supernatant liquid is again precipitated with absolute alcohol and filtered. The precipitate from both precipitations is dissolved in 10 c.c. of distilled water. This solution is again precipitated and filtered; the filtrate obtained from this is added to the first filtrate, and the whole evaporated to its original bulk $(10$ c.c.) at a temperature of $56^{\circ} \mathrm{C}$

The combined precipitates are dissolved in 10 c.c. of distilled water. The resulting solution contains the same amount of precipitate as the original 10 c.c. of old tuberculin. The solution of the precipitate is diluted with distilled water to form a 10 per cent. solution. If now 1 minim of this 10 per cent. solution of the precipitate is injected under the horny layer of the skin in three or four hours a small, raised, inflammatory area about half an inch in diameter appears around the site of injection in about 92 per cent. of all cases, both tubercular and apparently healthy individuals. This reaction, therefore, proves of no particular value in diagnosis.

The filtrate which has been evaporated to its original bulk is now taken and diluted with distilled water to form a 10 per cent. solution. If 1 minim of this solution be injected under the horny layer of the skin, a raised inflammatory area appears round the site of injection in from 12 to 18 huurs in known tuberculous individuals; no positive reaction has been noted in persons apparently free from tuberculous disease, such individuals being likewise free, according to their clinical histories, from any suspicion of tubercular affections.

In applying the $10 \mathrm{par}$ cent. solution of the filtrate for diagnostic purposes the procedure is as follows, A small area of the skin of the forearm is cleaned with soap spirit and rendercd tense, as one does when raccinating; the point of a hypodermic needle is then inserted obliquely between the horny and Malpighian layers, the aperture of the needle being turned towards the surface. One minim of the solution is then injected; a control of distilled water is injected in the same way. On withdrawing the needle some of the injected fluid escapes and is removed on a piece of cotton-wool. No dressing is required.

A positive reaction is seen to be as follows. Around the site of injection a raised area of inflammation and induration appears in from 12 to 18 hours. The inflammatory area in early cases of tuberculosis is usually nearly an inch in diameter, the edges gradually fading to normal skin. The area of induration is noted in the immediate vicinity of the needle prick. In more advanced cases the inflammatory area is smaller, usually about half an inch in diameter, the edges being more sharply defined. In these, too, the area of induration is well defined round the needle prick. In still more advanced cases the area of inflammation and induration is less well defined but recognisable, while in very advanced cases a reaction, if at all present, is only very slight. In early cases the reaction persists for three or four days, while in advanced cases it passes off in from 24 to 48 hours.

The test as described has up to the present been applied to 270 persons. Of these 191 were known to be suffering from tuberculous disease in various forms. Of these 191 tubercular cases the reaction was positive in 186 ; the 5 tubercular cases yielding a completely negative reaction were in the last stages of pulmonary tuberculosis and died within two weeks of the date of application of the test. In the remaining 79 negative cases no reaction whatever was noted, and in these great care was taken to exclude the possibility of a tuberculous infection past or present.

The reaction given by the filtrate has up to the present been found to have great value in assisting in the diagnosis of medical and surgical cases of tuberculosis, and especially so in cases of incipient phthisis otherwise in doubt.

Therapeutics. - A number of patients have been treated with the filtrate (for convenience termed T.F.), and so far the results have been very encouraging. The initial dose found to be most convenient is 0.001 c.c. This is increased every other day by from 0.0002 c.c. to 0.001 c.c. according to the reaction produced. It is easily borne and does not produce the untoward results so frequently given by other forms of tuberculin. The reactions by the patients are, as a rule, slight. There may be two hours after injection a slight rise of temperature, seldom more than $0.6^{\circ} \mathrm{F}$., and this usually becomes normal in ten hours. In some cases for about two or three hours a slight headache or feeling of malaise or pain near the site of injection is complained of, and this usually completely disappears in a few hours.

The remedy seems to have a beneficial effect on the temperature, which in about one-third of the cases treated was swinging and "hectic," became normal in a week, and remained so during the course of treatment. As a rule, over the site of the injection there appears a small swelling which passes off within 24 hours.

It has been customary to select the supraspinous fossa as the seat of injection. The skin is first painted with tinct. iodi, and the injection marle deep into the muscle with a platinum needle. Alternate shoulders are chosen to receive the injection every other day. Thirty cases of pulmonary tuberculosis have up to the present been treated with T.F. for two months and all have benefited thereby, as evidenced by the fall of temperature, increase in weight, disappearance or diminishing of the moist sounds in the chest, and lessening or disappearance of the cough, as well as a feeling of well-being expressed by the patients.

A typical case selected from the 30 cases treated is as follows. A male, aged 14 years. Admitted Sept. 9th, 1912 , suffering from pulmonary tuberculosis. The usual symptoms, cough, loss of weight, and night sweats, were complained of. On examination well-marked signs were discovered in the right upper and middle lobes, and some moist sounds and prolonged expiration in the left apex. Weight 5st. 10lb. temperature swinging from $97.2^{\circ}$ to $100^{\circ} \mathrm{F}$., with several reverses. Treatment was commenced on Sept. 12th ; within a week the temperature became normal and remained so ever since. Patient was given increasing closes of T.F. every other day. On Oct. 30th all the moist sounds in the chest had disappeared. Weight 6st. 10lb. Cough has quite disappeared and there are now no night sweats.

Union Hospital, Brarford. 